\title{
ELLIPTIC CURVES OVER THE PERFECT CLOSURE OF A FUNCTION FIELD
}

\author{
DRAGOS GHIOCA
}

\begin{abstract}
We prove that the group of rational points of a non-isotrivial elliptic curve defined over the perfect closure of a function field in one variable over a finite field is finiteley generated.
\end{abstract}

\section{INTRODUCTION}

The main ingredient for our result is a study of the Lehmer inequality for elliptic curves. The classical Lehmer conjecture (see [7], page 476) asserts that there is an absolute constant $C>0$ so that any algebraic number $\alpha$ that is not a root of unity satisfies the following inequality for its logarithmic height

$$
h(\alpha) \geq \frac{C}{[\mathbb{Q}(\alpha): \mathbb{Q}]} .
$$

A partial result towards this conjecture is obtained in [3. The analog of Lehmer conjecture for elliptic curves and abelian varieties asks for a good lower bound for the canonical height of a non-torsion point of the abelian variety. Also this question has been much studied (see [1] [2], [5], [9] [15]). As a consequence of our work, we obtain the following result.

Theorem 1.1. Let $K$ be a function field of transcendence degree 1 over $\mathbb{F}_{p}$. Let $E$ be a non-isotrivial elliptic curve defined over $K$. Then $E\left(K^{\mathrm{per}}\right)$ is finitely generated.

Using completely different methods, Minhyong Kim studied the set of rational points of non-isotrivial curves of genus at least two over the perfect closure of a function field in one variable over a finite field (see [6]).

Using the result of Theorem 1.1. Thomas Scanlon proved the full positive characteristic Mordell-Lang Conjecture for abelian varieties that are isogenous to a product of elliptic curves (see [1]).

Our plan is to prove first some general results about tame modules in Section 2 and then to prove Theorem 1.1 in Section 3 ,

\section{TAME MOdules}

Definition 2.1. Let $R$ be an integral domain and let $K$ be its field of fractions. If $M$ is an $R$-module, then by the rank of $M$, denoted $\operatorname{rk}(M)$, we mean the dimension of the $K$-vector space $M \otimes_{R} K$. We call $M$ a tame module if every finite rank submodule of $M$ is finitely generated.

Lemma 2.2. Let $R$ be a Dedekind domain and let $M$ be an $R$-module. Assume there exists a function $h: M \rightarrow \mathbb{R}_{\geq 0}$ satisfying the following properties

(i) (triangle inequality) $h(x \pm y) \leq h(x)+h(y)$, for every $x, y \in M$. 
(ii) if $x \in M_{\text {tor }}$, then $h(x)=0$.

(iii) there exists $c>0$ such that for each $x \notin M_{\text {tor }}, h(x)>c$.

(iv) there exists $a \in R \backslash\{0\}$ such that $R / a R$ is finite and for all $x \in M, h(a x) \geq 4 h(x)$.

If $M_{\mathrm{tor}}$ is finite, then $M$ is a tame $R$-module.

Proof. By the definition of a tame module, it suffices to assume that $M$ is a finite rank $R$-module and conclude that it is finitely generated.

Let $a \in R$ as in ( $i v)$ of Lemma 2.2. By Lemma 3 of [10, $M / a M$ is finite (here we use the assumption that $M_{\text {tor }}$ is finite). The following result is the key to the proof of Lemma 2.2,

Sublemma 2.3. For every $D>0$, there exists finitely many $x \in M$ such that $h(x) \leq D$.

Proof of Sublemma 2.3. If we suppose Sublemma 2.3 is not true, then we can define

$$
C=\inf \{D \mid \text { there exists infinitely many } x \in M \text { such that } h(x) \leq D\} .
$$

Properties $(i i)$ and $(i i i)$ and the finiteness of $M_{\text {tor }}$ yield $C \geq c>0$. By the definition of $C$, it must be that there exists an infinite sequence of elements $z_{n}$ of $M$ such that for every $n$,

$$
h\left(z_{n}\right)<\frac{3 C}{2} .
$$

Because $M / a M$ is finite, there exists a coset of $a M$ in $M$ containing infinitely many $z_{n}$ from the above sequence.

But if $k_{1} \neq k_{2}$ and $z_{k_{1}}$ and $z_{k_{2}}$ are in the same coset of $a M$ in $M$, then let $y \in M$ be such that $a y=z_{k_{1}}-z_{k_{2}}$. Using properties $(i v)$ and $(i)$, we get

$$
h(y) \leq \frac{h\left(z_{k_{1}}-z_{k_{2}}\right)}{4} \leq \frac{h\left(z_{k_{1}}\right)+h\left(z_{k_{2}}\right)}{4}<\frac{3 C}{4} .
$$

We can do this for any two elements of the sequence that lie in the same coset of $a M$ in $M$. Because there are infinitely many of them lying in the same coset, we can construct infinitely many elements $z \in M$ such that $h(z)<\frac{3 C}{4}$, contradicting the minimality of $C$.

From this point on, our proof of Lemma 2.2 follows the classical descent argument in the Mordell-Weil theorem (see [12).

Take coset representatives $y_{1}, \ldots, y_{k}$ for $a M$ in $M$. Define then

$$
B=\max _{i \in\{1, \ldots, k\}} h\left(y_{i}\right) \text {. }
$$

Consider the set $Z=\{x \in M \mid h(x) \leq B\}$, which is finite according to Sublemma 2.3. Let $N$ be the finitely generated $R$-submodule of $M$ which is spanned by $Z$.

We claim that $M=N$. If we suppose this is not the case, then by Sublemma 2.3] we can pick $y \in M-N$ which minimizes $h(y)$. Because $N$ contains all the coset representatives of $a M$ in $M$, we can find $i \in\{1, \ldots, k\}$ such that $y-y_{i} \in a M$. Let $x \in M$ be such that $y-y_{i}=a x$. Then $x \notin N$ because otherwise it would follow that $y \in N$ ( we already know $\left.y_{i} \in N\right)$. By our choice of $y$ and by properties $(i v)$ and $(i)$, we have

$$
h(y) \leq h(x) \leq \frac{h\left(y-y_{i}\right)}{4} \leq \frac{h(y)+h\left(y_{i}\right)}{4} \leq \frac{h(y)+B}{4} .
$$

This means that $h(y) \leq \frac{B}{3}<B$. This contradicts the fact that $y \notin N$ because $N$ contains all the elements $z \in M$ such that $h(z) \leq B$. This contradiction shows that indeed $M=N$ and so, $M$ is finitely generated. 
Corollary 2.4. Let $R$ be a Dedekind domain and let $M$ be a tame $R$-module.

(a) If $\operatorname{rk}(M)=\aleph_{0}$, then $M$ is a direct sum of a finite torsion submodule and a free submodule of rank $\aleph_{0}$.

(b) If $\operatorname{rk}(M)$ is finite and $R$ is a principal ideal domain, then $M$ is a direct sum of a finite torsion submodule and a free submodule of finite rank.

Proof. Part (a) of Corollary 2.4 is proved in Proposition 10 of 10 .

If $\operatorname{rk}(M)$ is finite and because $M$ is tame, we conclude that $M$ is finitely generated. Because $R$ is a principal ideal domain we get the result of part (b) of Corollary 2.4.

\section{ElLiptic CURVES}

The setting is the following: $K$ is a finitely generated field of transcendence degree 1 over $\mathbb{F}_{p}$ where $p$ is a prime as always. We fix an algebraic closure $K^{\text {alg }}$ of $K$. We denote by $\mathbb{F}_{p}^{\text {alg }}$ the algebraic closure of $\mathbb{F}_{p}$ inside $K^{\text {alg }}$.

Let $E$ be a non-isotrivial elliptic curve (i.e. $j(E) \notin \mathbb{F}_{p}^{\text {alg }}$ ) defined over $K$. Let $K^{\text {per }}$ be the perfect closure of $K$ inside $K^{\text {alg }}$. We will prove in Theorem 1.1 that $E\left(K^{\text {per }}\right)$ is finitely generated.

For every finite extension $L$ of $K$ we denote by $M_{L}$ the set of discrete valuations $v$ on $L$, normalized so that the value group of $v$ is $\mathbb{Z}$. For each $v \in M_{L}$ we denote by $f_{v}$ the degree of the residue field of $v$ over $\mathbb{F}_{p}$. If $P \in E(L)$ and $m \in \mathbb{Z}, m P$ represents the point on the elliptic curve obtained using the group law on $E$. We define a notion of height for the point $P \in E(L)$ with respect to the field $K$ (see Chapter VIII of [13] and Chapter III of [14])

$$
h_{K}(P)=\frac{-1}{[L: K]} \sum_{v \in M_{L}} f_{v} \min \{0, v(x(P))\} .
$$

Then we define the canonical height of $P$ with respect to $K$ as

$$
\hat{\mathrm{h}}_{E / K}(P)=\frac{1}{2} \lim _{n \rightarrow \infty} \frac{h_{K}\left(2^{n} P\right)}{4^{n}} .
$$

We also denote by $\Delta_{E / K}$ the divisor which is the minimal discriminant of $E$ with respect to the field $K$ (see Chapter VIII of [13]). By $\operatorname{deg}\left(\Delta_{E / K}\right)$ we denote the degree of the divisor $\Delta_{E / K}$ (computed with respect to $\mathbb{F}_{p}$ ). We denote by $g_{K}$ the genus of the function field $K$.

The following result is proved in [4].

Theorem 3.1 (Goldfeld-Szpiro). Let $E$ be an elliptic curve over a function field $K$ of one variable over a field in any characteristic. Let $\hat{\mathrm{h}}_{E / K}$ denote the canonical height on $E$ and let $\Delta_{E / K}$ be the minimal discriminant of $E$, both computed with respect to $K$. Then for every point $P \in E(K)$ which is not a torsion point:

$$
\hat{\mathrm{h}}_{E / K}(P) \geq 10^{-13} \operatorname{deg}\left(\Delta_{E / K}\right) \text { if } \operatorname{deg}\left(\Delta_{E / K}\right) \geq 24\left(g_{K}-1\right),
$$

and

$$
\hat{\mathrm{h}}_{E / K}(P) \geq 10^{-13-23 g} \operatorname{deg}\left(\Delta_{E / K}\right) \text { if } \operatorname{deg}\left(\Delta_{E / K}\right)<24\left(g_{K}-1\right) .
$$

We are ready to prove our result.

Proof of Theorem 1.1. We first observe that replacing $K$ by a finite extension does not affect the conclusion of the theorem. Thus, at the expense of replacing $K$ by a finite extension, we 
may assume $E$ is semi-stable over $K$ (the existence of such a finite extension is guaranteed by Proposition $5.4(a)$ of [13]).

As before, we let $\hat{\mathrm{h}}_{E / K}$ and $\Delta_{E / K}$ be the canonical height on $E$ and the minimal discriminant of $E$, respectively, computed with respect to $K$.

For every $n \geq 1$, we denote by $E^{\left(p^{n}\right)}$ the elliptic curve $F^{n}(E)$, where $F$ is the usual Frobenius (seen as morphism of varieties). Thus

$$
F^{n}: E\left(K^{1 / p^{n}}\right) \rightarrow E^{\left(p^{n}\right)}(K)
$$

is a bijection. Moreover, for every $P \in E\left(K^{1 / p}\right)$,

$$
p P=(V F)(P) \in V\left(E^{(p)}(K)\right) \subset E(K)
$$

where $V$ is the Verschiebung. Similarly, we get that

$$
p^{n} E\left(K^{1 / p^{n}}\right) \subset E(K) \text { for every } n \geq 1 \text {. }
$$

Thus $E\left(K^{\text {per }}\right)$ lies in the $p$-division hull of the $\mathbb{Z}$-module $E(K)$. Because $E(K)$ is finitely generated (by the Mordell-Weil theorem), we conclude that $E\left(K^{\text {per }}\right)$, as a $\mathbb{Z}$-module, has finite rank.

We will show next that the height function $\hat{\mathrm{h}}_{E / K}$ and $p \in \mathbb{Z}$ satisfy the properties $(i)$ - $(i v)$ of Lemma 2.2. Properties $(i)$ and $(i i)$ are well-known for $\hat{\mathrm{h}}_{E / K}$ and we also have the formula (see Chapter VIII of [13])

$$
\hat{\mathrm{h}}_{E / K}(p P)=p^{2} \hat{\mathrm{h}}_{E / K}(P) \geq 4 \hat{\mathrm{h}}(P) \text { for every } P \in E\left(K^{\mathrm{alg}}\right),
$$

which proves that property $(i v)$ of Lemma 2.2 holds.

Let $P$ be a non-torsion point of $E\left(K^{\text {per }}\right)$. Then $P \in E\left(K^{1 / p^{n}}\right)$ for some $n \geq 0$. Because $K^{1 / p^{n}}$ is isomorphic to $K$, they have the same genus, which we call it $g$. We denote by $\hat{\mathrm{h}}_{E / K^{1 / p^{n}}}$ and $\Delta_{E / K^{1 / p^{n}}}$ the canonical height on $E$ and the minimal discriminant of $E$, respectively, computed with respect to $K^{1 / p^{n}}$. Using Theorem 3.1. we conclude

$$
\hat{\mathrm{h}}_{E / K^{1 / p^{n}}}(P) \geq 10^{-13-23 g} \operatorname{deg}\left(\Delta_{E / K^{1 / p^{n}}}\right) .
$$

We have $\hat{\mathrm{h}}_{E / K^{1 / p^{n}}}(P)=\left[K^{1 / p^{n}}: K\right] \hat{\mathrm{h}}_{E / K}(P)=p^{n} \hat{\mathrm{h}}_{E / K}(P)$. Similarly, using the proof of Proposition $5.4(b)$ of [13],

$$
\operatorname{deg}\left(\Delta_{E / K^{1 / p^{n}}}\right)=p^{n} \operatorname{deg}\left(\Delta_{E / K}\right) .
$$

We conclude that for every $P \in E\left(K^{\text {per }}\right)$,

$$
\hat{\mathrm{h}}_{E / K}(P) \geq 10^{-13-23 g} \operatorname{deg}\left(\Delta_{E / K}\right) \text {. }
$$

Because $E$ is non-isotrivial, $\Delta_{E / K} \neq 0$ and so, $\operatorname{deg}\left(\Delta_{E / K}\right) \geq 1$. We conclude

$$
\hat{\mathrm{h}}_{E / K}(P) \geq 10^{-13-23 g} \text {. }
$$

Inequality (8) shows that property (iii) of Lemma 2.2 holds for $\hat{\mathrm{h}}_{E / K}$. Thus properties (i)-(iv) of Lemma 2.2 hold for $\hat{\mathrm{h}}_{E / K}$ and $p \in \mathbb{Z}$.

We show that $E_{\text {tor }}\left(K^{\text {per }}\right)$ is finite. Equation (5) shows that the prime-to- $p$-torsion of $E\left(K^{\text {per }}\right)$ equals the prime-to- $p$-torsion of $E(K)$; thus the prime-to-p-torsion of $E\left(K^{\text {per }}\right)$ is finite. If there exists infinite $p$-power torsion in $E\left(K^{\text {per }}\right)$, equation (3) yields that we have arbitrarily large $p$-power torsion in the family of elliptic curves $E^{\left(p^{n}\right)}$ over $K$. But this contradicts standard results on uniform boundedness for the torsion of elliptic curves over 
function fields, as established in [8] (actually, [8] proves a uniform boundedness of the entire torsion of elliptic curves over a fixed function field; thus including the prime-to- $p$-torsion). Hence $E_{\text {tor }}\left(K^{\text {per }}\right)$ is finite.

Because all the hypothesis of Lemma 2.2 hold, we conclude that $E\left(K^{\text {per }}\right)$ is tame. Because $\operatorname{rk}\left(E\left(K^{\text {per }}\right)\right)$ is finite we conclude by Corollary 2.4 $(b)$ that $E\left(K^{\text {per }}\right)$ is a direct sum of a finite torsion submodule and a free submodule of finite rank.

Remark 3.2. It is absolutely crucial in Theorem 1.1 that $E$ is non-isotrivial. Theorem 1.1 fails in the isotrivial case, i.e. there exists no $n \geq 0$ such that $E\left(K^{\text {per }}\right)=E\left(K^{1 / p^{n}}\right)$. Indeed, if $E$ is defined by $y^{2}=x^{3}+x(p>2), K=\mathbb{F}_{p}\left(t,\left(t^{3}+t\right)^{\frac{1}{2}}\right)$ and $P=\left(t,\left(t^{3}+t\right)^{\frac{1}{2}}\right)$, then $F^{-n} P \in E\left(K^{1 / p^{n}}\right) \backslash E\left(K^{1 / p^{n-1}}\right)$, for every $n \geq 1$. So, $E\left(K^{\text {per }}\right)$ is not finitely generated in this case (and we can get a similar example also for the case $p=2$ ).

\section{REFERENCES}

[1] M. Baker, J. Silverman, A lower bound for the canonical height on abelian varieties over abelian extensions. Math. Res. Lett. 11 (2004), no. 2-3, 377-396.

[2] S. David, J. Silverman, Minoration de la hauteur de Néron-Tate sur les variétés abéliennes de type C. M. (French) [Lower bound for the Néron-Tate height on abelian varieties of CM type] J. Reine Angew. Math. 529 (2000), 1-74.

[3] E. Dobrowolski, On a question of Lehmer and the number of irreducible factors of a polynomial. Acta Arith. 34, no. 4, 391-401, (1979).

[4] D. Goldfeld, L. Szpiro, Bounds for the order of the Tate-Shafarevich group Special issue in honour of Frans Oort. Compositio Math. 97 (1995), no. 1-2, 71-87

[5] M. Hindry, J. Silverman, On Lehmer's conjecture for elliptic curves. Séminaire de Théorie des Nombres, Paris 1988-1989, 103-116, Progr. Math., No. 91, Birkhäuser Boston, Boston, MA, 1990.

[6] Minhyong Kim, Purely inseparable points on curves of higher genus. Math.Res.Lett. 4 (1997), no. 5, 663-666.

[7] D. H. Lehmer, Factorization of certain cyclotomic polynomials, Ann. of Math. (2) 34 (1933), no. 3, 461-479.

[8] Martin Levin, On the group of rational points on elliptic curves over function fields, Amer.J.Math 90, $1968,456-462$

[9] D.W. Masser, Counting points of small height on elliptic curves. Bull. Soc. Math. France 117 (1989), no. $2,247-265$.

[10] B. Poonen, Local height functions and the Mordell-Weil theorem for Drinfeld modules, Compositio Mathematica 97 (1995), 349-368.

[11] T. Scanlon, Positive characteristic Manin-Mumford theorem. preprint, 2003, available online at

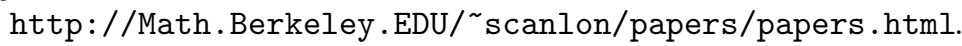

[12] Jean-Pierre Serre, Lectures on the Mordell-Weil theorem. Translated from the French and edited by Martin Brown from notes by Michel Waldschmidt. Aspects of Mathematics, E15. Friedr. Vieweg \& Sohn, Braunschweig, 1989. $\mathrm{x}+218$ pp.

[13] Joseph Silverman, The arithmetic of elliptic curves. Graduate Texts in Mathematics, 106. SpringerVerlag, New York, 1986. xii+400 pp.

[14] Joseph Silverman, Advanced topics in the arithmetic of elliptic curves. Graduate Texts in Mathematics, 151. Springer-Verlag, New York, 1994. xiv +525 pp.

[15] J. Silverman, A lower bound for the canonical height on elliptic curves over abelian extensions. J. Number Theory 104 (2004), no. 2, 353-372. 